\title{
GESTIÓN DE RIESGOS DEL PATRIMONIO CULTURAL: ALCANCES PARA EL PATRIMONIO HISTÓRICO INMUEBLE ${ }^{[*]}$
}

\author{
RISK MANAGEMENT OF CULTURAL HERITAGE: OUTLOOKS FOR HISTORICAL HERITAGE
}

\author{
CLARY DIANA SANTANDER CJUNO ${ }^{[*]}$ \\ Fecha de recepción: 03 de abril de 2017 \\ Fecha de aprobación: 20 de mayo de 2017
}

\section{RESUMEN}

El presente artículo relaciona conceptos básicos de la gestión de patrimonio y la gestión de riesgos del patrimonio cultural (GRPC). Para abordar este tema se parte por delinear ciertos conceptos cruciales sobre patrimonio cultural, pues la teoría formulada al respecto es dinámica, cambiante y resulta de gran importancia para la sociedad actual. Para abordar la temática central se han utilizado conceptos vinculados con los riesgos, el patrimonio cultural y su gestión, para posteriormente integrarlos con mayor detalle al enfoque aquí abordado. Finalmente, se han incluido los aspectos más relevantes de la metodología desarrollada por el Instituto Canadiense de Conservación (CCI), el Centro Internacional de Estudios para la Conservación y la Restauración de los Bienes Culturales (ICCROM), y la Agencia de los Países Bajos para el Patrimonio Cultural (RCE). La gestión de riesgo abarca la evaluación, análisis, propuestas, planes y otros aspectos vinculados a la práctica de una organización pública/privada, con el objetivo de minimizar el riesgo en el momento de la toma de decisiones sobre la gestión del patrimonio cultural.

\section{PALABRAS CLAVE}

Gestión de riesgos, patrimonio cultural, patrimonio histórico inmueble

\section{ABSTRACT}

This article connects basic concepts to heritage management and risk management of cultural heritage. Thus, to tackle this subject, it starts with a picture of the concepts of cultural heritage, since the theory about it is dynamic, changing, and very important for contemporary society. In order to address the central topic, concepts related to risks, cultural heritage and their management have been employed, to then integrate them with greater detail to the approach studied here. Finally, the most relevant aspects of the methodology developed by the Canadian Conservation Institute (ICC), the International Center for the Study of the Preservation and Restoration of Cultural Property (ICCROM) and the Cultural Heritage Agency of the Netherlands (RCE) have been included. Risk management encompasses the evaluation, analysis, proposals, plans and other aspects related to the practice of a public/private organization in order to minimize risk when decision making about cultural heritage management occurs.

\section{KEYWORDS}

Risk management, cultural heritage, historical heritage assets

(*) El presente artículo forma parte del plan de tesis titulado La Valoración de Riesgos para el Patrimonio Cultural Inmueble de un Poblado Histórico como Instrumento de Gestión del Patrimonio Cultural. Estudio y Análisis del Caso de la Zona Monumental de Chinchero-Cusco, propuesto en diciembre de 2016 para la obtención del grado académico de magíster en Ciencias en Conservación y Gestión del Patrimonio Edificado en la Unidad de Posgrado de la Facultad de Arquitectura, Urbanismo y Artes de la Universidad Nacional de Ingeniería, bajo la dirección del MSc. Arq. José Hayakawa Casas.

(**) Arquitecta por la Universidad Nacional de San Antonio Abad del Cusco (2005). Estudios de maestría en Ciencias en Conservación y Gestión del Patrimonio Edificado en la Universidad Nacional de Ingeniería. Con cursos en Gestión de Riesgos para el Patrimonio Cultural del Centro Internacional para el Estudio de la Preservación y Restauración del Patrimonio Cultural, y la Dirección de Bibliotecas, Archivos y Museos de Chile (Chile, 2013); de Preservación de Conjuntos y Centros Históricos del Centro Internacional para la Conservación del Patrimonio (Argentina, 2012). Ha trabajado en el Centro Guamán Poma de Ayala (Cusco), el Instituto Nacional de Cultura (Lima) y el Ministerio de Cultura de Perú (Lima). Actualmente, se desempeña como consultora independiente. Contacto: cdianas@icloud.com 
La motivación por investigar sobre la gestión de riesgo orientada a la preservación del patrimonio cultural surge de la búsqueda de alternativas económicas y técnicas viables para su adecuada gestión. En el presente caso, la investigación está enfocada en el patrimonio histórico inmueble en particular, debido a dos factores: un número importante de bienes de conforman ese patrimonio en el territorio peruano, y se requiere de criterios de mayor amplitud académica para ser intervenirlos.

En relación con el patrimonio en general, la Organización de las Naciones Unidas para la Educación, la Ciencia y la Cultura (UNESCO) señala que es "el legado que recibimos del pasado, lo que vivimos en el presente y lo que trasmitimos a las futuras generaciones" $(2008$, p. 5). Es decir, puede entenderse como el legado de nuestros antepasados. Además, como Gonzáles-Varas señala, "el patrimonio cultural es asimismo elemento fundamental para la construcción de identidades colectivas, en sus diversas escalas local o regional, nacional, internacional o global, (...) [por lo tanto, es] (...) todo aquello que reconocemos valoramos y deseamos conservar de la cultura (...)" (2015, pp. 2225), y su selección dependerá de las valoraciones sociales, ideológicas e intelectuales que se realicen a través del tiempo; es decir, el patrimonio cultural es dinámico y el valor que se le otorga está directamente relacionado con el grupo social que lo reconoce.

Dado que la naturaleza del patrimonio cultural es diversa, existen distintas categorías que permiten clasificarlo. Según su origen, es posible identificar dos grandes grupos: material e inmaterial, también denominados tangible e intangible. El patrimonio cultural material es esencialmente aquel que tiene una existencia física; por otro lado, el patrimonio cultural inmaterial es aquel que se encuentra en prácticas: tradiciones, costumbres, expresiones, entre otros. En este estudio es relevante el patrimonio cultural material, que a su vez se divide en dos categorías: mueble e inmueble. La primera reúne a bienes que pueden trasladarse, mientras que la segunda se aplica a bienes que no pueden trasladarse, como un área urbana o una edificación.

En el Perú, el patrimonio cultural inmueble de data virreinal y republicana, de acuerdo con el portal web de Infocultura del Ministerio de Cultura (2016), involucra alrededor de 5,125 bienes. Estos bienes cuentan con la denominación Bien Integrante del Patrimonio Cultural de la Nación, conforme lo señala la Ley № 28296, Ley General del Patrimonio Cultural de la Nación. Según dicha ley, pueden considerarse bienes inmuebles materiales los edificios, obras de infraestructura, ambientes y conjuntos monumentales, centros históricos, y demás construcciones o evidencias. Entre los bienes declarados, de acuerdo con el portal web del Ministerio de Cultura (2016), se encuentran registradas las siguientes categorías: ambiente monumental (4 bienes), ambiente urbano monumental (417), inmueble de valor monumental (64), inmueble identificado para su declaración (173), monumento (4,394), paisaje cultural arqueológico e histórico (1), valor urbanístico de entorno (1), zona histórico monumental (5), zona monumental (64) y zona paisajística de valor monumental (2).

\section{Conceptos importantes}

Según Mirabile, "es común confundir los significados de los términos 'agente de deterioro', 'amenaza', 'peligro' y 'riesgo' con los procesos y mecanismos que causan el deterioro" (citado en Mattos \& Gonçalves 2015, p. 145). Los mismos autores, al referirse a la aplicación de la terminología de gestión a la conservación del patrimonio cultural, señalan lo siguiente:

A pesar del conocimiento sólido producido por la Ciencia de la Conservación, a partir de la segunda mitad del siglo XX, los profesionales y las instituciones que se ocupan de la conservación de los bienes culturales tienen como costumbre aplicar normas, directrices y prácticas sin un sentido claro de cómo establecer prioridades o considerar los costos involucrados con el fin de hacer un mejor uso de los recursos disponibles. También hay que señalar que la terminología utilizada en el área aún no ha sido estandarizada. (2015, p. 144) 
Así, para el caso estudiado resulta de gran importancia y utilidad precisar ciertos conceptos estratégicos que permiten construir una mirada más sistemática y fundamentada de la gestión de riesgos del patrimonio cultural.

\section{Riesgo y patrimonio cultural}

Según la Organización de las Naciones Unidas, el riesgo es "La combinación de la probabilidad de que se produzca un evento y sus consecuencias negativas" (2009, p. 29). En la literatura científica, se entiende como riesgo al producto de los factores vinculados a una amenaza y aquellos referidos a la vulnerabilidad. Es decir, "para que exista un riesgo debe existir una amenaza (por ejemplo, un terremoto, un incendio) que interactúa con una vulnerabilidad (constructiva, arquitectónica, social) en un determinado contexto espacial y temporal" (Jorquera, 2014, p. 56). En muchos casos los riesgos no pueden eliminarse, por lo que son de vital importancia las decisiones que se tomen para disminuir, controlar o mitigar las posibles consecuencias de una amenaza. Así, la gestión del riesgo constituye "el enfoque y la práctica sistemática de gestionar la incertidumbre para minimizar los daños y las pérdidas potenciales" (UNISDR, 2009, p. 18). Por lo tanto, abarca la evaluación, el análisis, la elaboración de propuestas y planes, y otros aspectos vinculados a la práctica de una organización que tiene como objetivo minimizar el riesgo mediante una adecuada toma de decisiones. En cuanto al concepto de riesgo vinculado específicamente al patrimonio cultural, ICCROM (2016) propone lo siguiente:

Muchas cosas puede que tengan un impacto negativo en las colecciones patrimoniales, edificios, monumentos, sitios, y sobre nuestros objetivos relativos a su uso y preservación. El impacto de los riesgos en este caso se expresa en términos de pérdida de valor esperada para el patrimonio cultural.

Los tipos de riesgos para nuestro patrimonio cultural varían de extraordinarios y eventos frecuentes (como terremotos, inundaciones, incendios y conflictos armados) a procesos continuos y acumulativos (tales como procesos químicos, físicos o degradación). El resultado es la pérdida de valor del patrimonio cultural. Por ejemplo, si una casa histórica se incendia hay una pérdida importante o total de valor del edificio y su contenido. Cuando los objetos frágiles de una colección del museo se rompen durante un terremoto hay una pérdida de valor en esa colección. (...) Los gestores del patrimonio y sus custodios deben entender bien estos riesgos para tomar buenas decisiones sobre la protección del patrimonio (para futuras generaciones)... (p. 11)

\section{Gestión de riesgo}

La gestión de riesgo consiste en "el enfoque y la práctica sistemática de gestionar la incertidumbre para minimizar los daños y las pérdidas potenciales" (UNISDR, 2009, p. 18). Por lo tanto, tal práctica abarca la evaluación, el análisis, la elaboración de propuestas y planes, y otros aspectos vinculados a la toma de decisiones en el interior de una organización cuya labor consiste en minimizar potenciales daños y pérdidas. Ampliando lo señalado, ICCROM (2016) plantea lo siguiente:

La gestión de riesgos es todo lo que hacemos para entender y hacer frente a posibles impactos negativos en nuestros objetivos. Incluye la identificación, análisis y priorización (lo llamamos evaluación) de los riesgos. Entonces tomamos medidas para "tratar" los riesgos, es decir, para evitar, eliminar o reducir los riesgos que consideramos inaceptables. También podemos transferir esos riesgos a otros. Por ejemplo, cuando aseguramos nuestras colecciones, transferimos el riesgo de robo o daño a la compañía de seguros (por una tarifa).

Si uno o más riesgos son evaluados como aceptables, no necesitamos hacer nada al respecto. (...)

Es importante recordar que la gestión de riesgos es un proceso continuo. Tenemos que seguir vigilando los riesgos y ajustando nuestras acciones para asegurar que los impactos negativos en nuestros objetivos serán minimizados. 
Utilizado en campos como la salud pública, el medio ambiente y la tecnología, la gestión de riesgos es una herramienta esencial para que el gobierno y la industria alcancen sus objetivos de una manera más controlada y exitosa. (p. 14).

En paralelo, es importante conocer la definición de gestión de riesgo que maneja operativamente el Gobierno peruano. La Ley № 29664, ley que crea el Sistema Nacional de Gestión del Riesgo de Desastres (SINAGERD), publicada en el diario oficial El Peruano el 19 de febrero de 2011, señala lo siguiente en el artículo $3^{\circ}$ :

La Gestión del Riesgo de Desastres es un proceso social cuyo fin último es la prevención, la reducción, y el control permanente de los factores de riesgo de desastres en la sociedad, así como la adecuada preparación y respuesta ante situaciones de desastre, considerando las políticas nacionales con especial énfasis en aquellas relativas a materia económica, ambiental, de seguridad, defensa nacional y territorial de manera sostenible.

La Gestión del Riesgo de Desastres está basada en la investigación científica y de registro de informaciones, y orienta las políticas, estrategias y acciones en todos los niveles de gobierno y de la sociedad con la finalidad de proteger la vida de la población y el patrimonio de las personas y del Estado.

De ello puede inferirse que el Patrimonio Cultural de la Nación, al ser patrimonio del Estado, está considerando dentro de los planes de gestión de desastres.

\section{Gestión del patrimonio cultural}

Según Tello, "A través de la 'patrimonialización generalizada'se traslada el pasado hacia el presente, con el fin de revivirlo y gestionarlo para el futuro, es decir, de salvaguardarlo. En ese sentido los bienes patrimoniales se convierten en recursos susceptibles de gestión" (2002, p. 11). Complementando lo señalado, Ballart indica que la gestión patrimonial constituye un

...conjunto de actuaciones programadas con el objetivo de conseguir una óptima conservación de los bienes patrimoniales y un uso de estos adecuado a las exigencias sociales contemporáneas (...). La gestión del patrimonio histórico tiene como punto de partida la conservación de determinados objetos especialmente apreciados, producidos por la actividad humana en un pasado más o menos lejano, que han perdurado hasta el presente. (2001, pp. 15-16)

Al respecto, se puede acotar que la gestión del patrimonio cultural constituye "una alternativa estratégica y contemporánea que tienen nuestras sociedades para lidiar con el rol cada vez más importante y complejo que cumple la cultura" (Hayakawa, 2009 , p. 84). Asimismo, al referirse a las principales funciones de las instituciones responsables de la gestión del patrimonio, Ballart señala las siguientes: "1. Identificar, recuperar y reunir grupo de objetos y colecciones. 2. Documentarlos. 3. Conservarlos. 4. Estudiarlos. 5. Presentarlo y exponerlos públicamente. 6. Interpretarlos o explicarlos" (2001, p. 23). Acorde a tales planteamientos, UNESCO señala en relación con la gestión cultural lo siguiente:

Una gestión correcta del potencial de desarrollo del patrimonio cultural exige un enfoque que haga hincapié en la sostenibilidad. A su vez la sostenibilidad requiere encontrar el justo equilibrio entre sacar provecho del patrimonio cultural hoy y preservar su 'riqueza frágil' para las generaciones futuras. (2014b, p. 132)

Además, la misma organización señala que la gestión se compone de procesos, los cuales buscan "el uso juicioso de los medios para alcanzar un fin" (2014b, p. 25). Asimismo, utiliza las expresiones sistema de gestión y sistema de gestión del patrimonio cultural:

...'sistema de gestión' puede definirse como una serie de procesos que, en conjunto, proporcionan una secuencia de resultados, algunos de los cuales se reintroducen en el sistema para 
crear un espiral ascendente de mejoras continuas del sistema, sus acciones y sus logros (...) Un 'sistema de gestión del patrimonio cultural' ayuda a conservar y gestionar un determinado bien o grupo de bienes de un modo que proteja los valores del patrimonio, y en particular el VUE [referido al Valor Único Excepcional] si se trata de un bien del Patrimonio Mundial, y siempre que sea posible, promueve la obtención de beneficios sociales, económicos y ambientales más allá de los límites del bien. Esta intervención más amplia es un factor disuasivo en el empleo de prácticas nocivas para el patrimonio cultural y facilita la identificación y promoción de los valores patrimoniales de un bien. Además, confiera una función constructiva al patrimonio cultural realzando el desarrollo humano lo que, a la larga, es beneficioso porque aumenta la sostenibilidad del propio patrimonio cultural... (2014b p. 25-26)

Dentro de este marco, la UNESCO (2014c) propone tres indicadores de la gestión que se encuentran interrelacionados:

\begin{abstract}
...'Registros e Inscripciones' ofrece una aproximación al grado en que los recursos patrimoniales de un país se reconocen como valiosos y merecedores de protección oficial para su salvaguardia. Partiendo de la premisa de que las listas e inventarios del patrimonio cultural se establecen 'con miras a la conservación y la salvaguardia', registrar e inventariar no son ejercicios abstractos, sino acciones instrumentales necesarias que dan una medida del grado de voluntad política en la materia. Así, el nivel de compromiso en la elaboración de registros e inscripciones, incluida su actualización periódica, ofrece una buena indicación estructural del grado de prioridad que se concede al patrimonio.
\end{abstract}

(...) 'Protección, Salvaguardia y Gestión' pone de relieve la medida en que las autoridades públicas aseguran la conservación, la valorización y la gestión sostenible del patrimonio; la imprescindible formación y fortalecimiento de capacidades de los principales interesados; y la participación activa de las comunidades concernidas. En efecto, para que la voluntad y la intención públicas de cuidar del patrimonio - reflejadas en los registros e inscripciones - se traduzcan en protección, salvaguardia y valorización genuinas será necesario que se aprueben y apliquen políticas y medidas concretas.

(...) 'Transmisión y Movilización de Apoyos' observa los esfuerzos desplegados a fin de sensibilizar a las comunidades y ciudadanos y acrecentar su conocimiento sobre el valor y el sentido del patrimonio. Atiende también a las inversiones continuadas en promoción del patrimonio con la implicación del sector privado y de la sociedad civil, de manera que el mensaje de su valor e importancia se transmita a las generaciones futuras. (p. 133)

\title{
Gestión de Riesgos del Patrimonio Cultural (GRPC)
}

El CCI, el ICCROM y la RCE han desarrollado una propuesta metodológica que se enfoca en la conservación preventiva desde la gestión de riesgos. La propuesta metodológica parte de las bases técnicas desarrolladas en la norma internacional ISO 31000 y en la Norma Técnica Colombiana para la Gestión del Riesgo NTC-ISO 31000.

En primer término, es importante tener presente que la gestión del riesgo en general es un concepto que puede aplicarse a distintos ámbitos, como la agricultura, la economía, entre otros. La Norma Técnica Colombiana de Gestión del Riesgo NTC 5254 la define como la "cultura, procesos y estructuras que se dirigen hacia la gestión eficaz de las oportunidades potenciales y los efectos adversos" (2004, p. 4).

El riesgo puede ser definido como la "posibilidad de que suceda algo que tendrá impacto en los objetivos. Se mide en términos de consecuencias y posibilidad de ocurrencia" (Instituto Colombiano de Normas Técnicas y Certificación, 2004, p. 3). Por otro lado, la ONU define el riesgo como "la combinación de la probabilidad de que se produzca un evento y sus consecuencias negativas" (UNISDR, 2009, p. 29). A partir de tales planteamientos, la gestión del riesgo del patrimonio puede considerarse un nuevo enfoque en el ámbito de la conservación preventiva, cuyo objetivo es analizar la potencial pérdida de valores del patrimonio cultural ante posibles riesgos, a fin de que los 
gestores del patrimonio puedan tomar decisiones para su preservación y salvaguarda. La normativa internacional vinculada a la gestión de riesgos ha establecido un determinado proceso, el cual consiste en la "aplicación sistemática de políticas de gestión, procedimientos y prácticas, a las tareas de establecimiento del contexto, identificación, análisis, evaluación, tratamiento, monitoreo y comunicación del riesgo" (Instituto Colombiano de Normas Técnicas y Certificación, 2004, p. 5).

Al respecto, Michalski \& Pedersoli (2011) señalan que el proceso de la gestión de riesgos, estructurado según la norma técnica Australian/New Zealand Standard for Risk Management, consta de cinco pasos secuenciales (establecer el contexto, e identificar los riesgos, analizarlos, evaluarlos y tratarlos), y dos pasos continuos (comunicación y consulta, y monitoreo y revisión). Entre los pasos señalados son cruciales aquellos vinculados a la identificación, análisis y evaluación de riesgos, aquellos que en conjunto, según señalan los autores, constituyen la valoración de riesgos (Figura 1).

Según señala la NTC 5254, los elementos principales que conforman el proceso de la gestión de riesgos son los siguientes:

1. Establecer el contexto estratégico, organizacional y de gestión en el cual ocurrirá el resto del proceso. Es conveniente establecer los criterios que se emplearán para evaluar el riesgo, así como definir la estructura del análisis.

2. Identificar los riesgos. Identificar qué, por qué y cómo pueden surgir elementos como base para el análisis posterior.

3. Determinar los controles existentes y analizar los riesgos en términos de consecuencia y posibilidad en el contexto de estos. El análisis debe considerar la gama de consecuencias potenciales y la probabilidad de que estas ocurran. Se pueden combinar consecuencia y posibilidad para determinar un nivel estimado de riesgo.

4. Evaluar los riesgos. Comparar los niveles estimados de riesgo con los criterios preestablecidos, para clasificar los riesgos e identificar las prioridades de gestión. Si se determina que los niveles de riesgo son bajos, puede que no se requiera tratamiento.

5. Tratar los riesgos. Aceptar y monitorear los riesgos de baja prioridad. En cuanto a los demás riesgos, corresponde desarrollar e implementar un plan de gestión específico que considere el suministro de recursos.

6. Monitorear y revisar el desempeño del sistema de gestión del riesgo y los cambios que pudieran afectarlo.

7. Comunicar y consultar con las partes interesadas, internas y externas, según sea apropiado, en cada etapa del proceso de gestión del riesgo, con relación al proceso en conjunto. (Instituto Colombiano de Normas Técnicas y Certificación, 2004)

\section{Valoración de riesgos que afectan al patrimonio cultural}

La valoración de riesgos constituye el "proceso general de análisis del riesgo y evaluación del riesgo" (Instituto Colombiano de Normas Técnicas y Certificación., 2004, p. 3). Al respecto, Michalski \& Pedersoli (2011) señalan que se trata del proceso central de la gestión de riesgos, que consta de tres pasos:

- Identificación de riesgos. Es uno de los pasos esenciales de la gestión de riesgo y consiste en el "proceso para determinar lo que puede suceder, por qué y cómo" (NTC 5254, 2004, p. 4). Para identificar un riesgo, según señalan Michalski \& Pedersoli (2011), se combinan dos tipos identificables: los vulnerables a un agente específico y los expuestos a ese agente en particular. Para realizar la identificación y organización de los riesgos en patrimonio cultural, se parte de los agentes de deterioro o pérdida desarrollados por el CCl. 


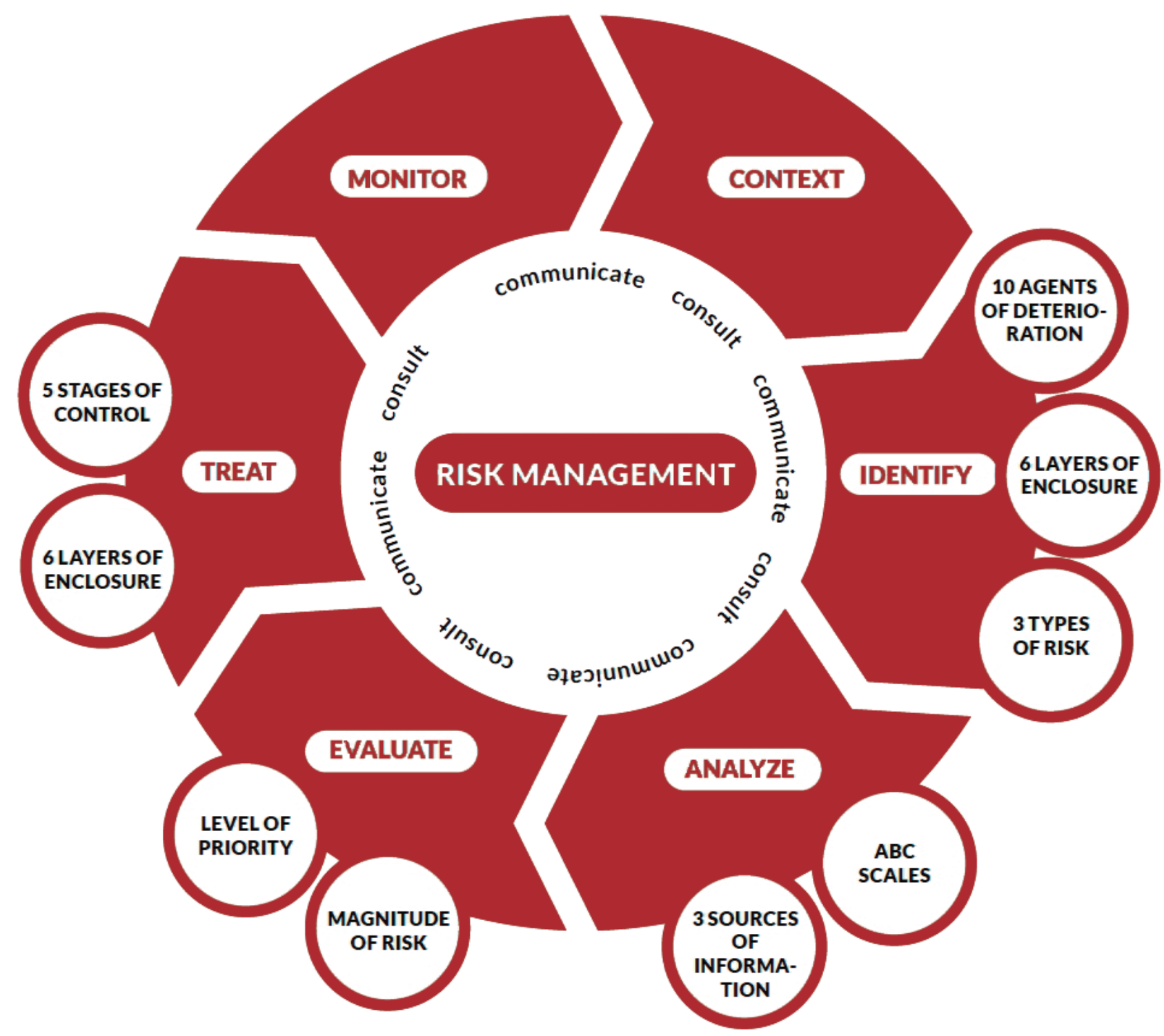

- Análisis de riesgo. El análisis del riesgo es el "uso sistemático de la información disponible, para determinar la frecuencia con la que pueden ocurrir eventos especificados y la magnitud de sus consecuencias" (NTC 5254, 2004, p. 3). Es decir, es la sumatoria de la frecuencia y la consecuencia. La frecuencia constituye un único "componente que mezcla dos tipos de momentos: eventos y procesos", mientras que la consecuencia se divide en "pérdida a futuro de cada objeto afecto (...) y (...) valor actual de todos los elementos afectados" (Michalski \& Pedersoli, 2011, p. 54), es decir, la relación entre la pérdida de valor de cada objeto (inmueble) y la pérdida de valor en relación con el conjunto. El producto de estos valores genera la cuantificación del riesgo por agente de deterioro.

- Evaluación del riesgo. Es el "proceso usado para determinar las prioridades de gestión mediante la comparación del nivel de riesgo contra normas predeterminadas, niveles de riesgo objeto u otros criterios." (NTC 5254, 2004, p. 4). Incluye otros aspectos, según señalan Michalski \& Pedersoli: los “riesgos son evaluados en contraposición a criterios que no
Figura 1. Ciclo de gestión de riesgos basado en el Standard AS/NZ 4360:2004 de Australia y Nueva Zelanda

Fuente: A Guide to Risk Management of Cultural Heritage (p. 15), por ICCROM, 2016, Sharjah, United Arab Emirates: Autor. 
sólo incluyen magnitud. Estos criterio abarcan: el nivel de incertidumbre asociada a cada riesgo; el grado de convergencia entre riesgos; y el contexto..." (2011, p. 80).

\section{Los diez agentes de deterioro y pérdida}

EI CCI ha desarrollado un enfoque para identificar amenazas denominado 10 agentes de deterioro y pérdida. Al respecto, Michalski \& Pedersoli (2011) señalan que tal enfoque corresponde a un esquema de clasificación de las posibles amenazas que pueden afectar a un bien patrimonial. Esta clasificación constituye un marco de referencia básico para organizar los riesgos al identificarlos; además, permite pensar cómo tales agentes actúan en los bienes, así como analizar sus causas y efectos (Figura 2).

1. Fuerzas físicas. Según señala el Marcon (2009), la fuerza física puede dañar directamente a los objetos, provocando rotación, deformación, tensión y presión; también puede hacerlo indirectamente, al generar choque entre estos o sus partes. El daño ocasionado por dicha fuerza puede implicar desde pequeñas fisuras, imperceptibles y diminutas pérdidas, hasta efectos a gran escala, tales como el aplastamiento de objetos, el hundimiento de suelos y, en casos extremos, la destrucción de construcciones enteras.

2. Robo y actos vandálicos. El robo constituye la extracción oportunista, intencionada o premeditada ilegal de un bien. El vandalismo consiste en infligir daño de forma intencional o premeditada a un bien, lo cual puede provocar su destrucción o desfiguración. En su mayoría, los actos de vandalismo ocurren ocasionalmente, por ejemplo las pintas en paredes o esculturas urbanas. Tremain (2009a), respecto de este agente, define lo siguiente:

Robo

El robo consiste en la extracción oportunista, intencionada o premeditadamente ilegal de un bien. La mayoría de los robos (...) tienden a ser sucesos aislados y no ejecutados por profesionales.

Vandalismo

Consiste en infligir daño de forma intencional o premeditada a un bien, que puede provocar su destrucción o desfiguración. Muy pocos actos de vandalismo resultan ser premeditados; en su mayoría son ocasionales, realizados también por los visitantes, por personas mentalmente inestables, o por visitantes que se encuentran bajo la influencia del alcohol o las drogas... (p. 10)

3. Fuego. Al respecto, Steward (2009) asevera lo siguiente:

El fuego es el estado de combustión resultante de una reacción química que requiere la combinación apropiada de tres elementos - una fuente de combustible (algo que se queme), oxígeno (un componente del aire) y una fuente de ignición, como calor o una chispa- para que se inicie y desarrolle. A este proceso se denomina con frecuencia 'Triángulo del Fuego', (...). La extinción del fuego generalmente requiere eliminar al menos uno de estos tres elementos.

(...)

Debido a que el fuego puede crecer y propagarse rápidamente, es importante detectarlo y extinguirlo en la etapa más temprana posible para así reducir el riesgo de daño severo, lesiones o pérdidas. (p. 11)

4. Agua. Corresponde a las afectaciones provocadas por el agua en forma líquida, como la filtración por capilaridad, o por eventos naturales como tormentas, inundaciones y huracanes. Puede ocasionar la pérdida parcial o total de un bien. Según señala Tremain (2009b), el daño ocasionado por el agua puede responder a distintos factores:

Puede originarse a partir de eventos naturales, amenazas tecnológicas o fallas mecánicas. Sin embargo, la mayoría de los problemas relacionados con el agua (...) son resultado de acci- 


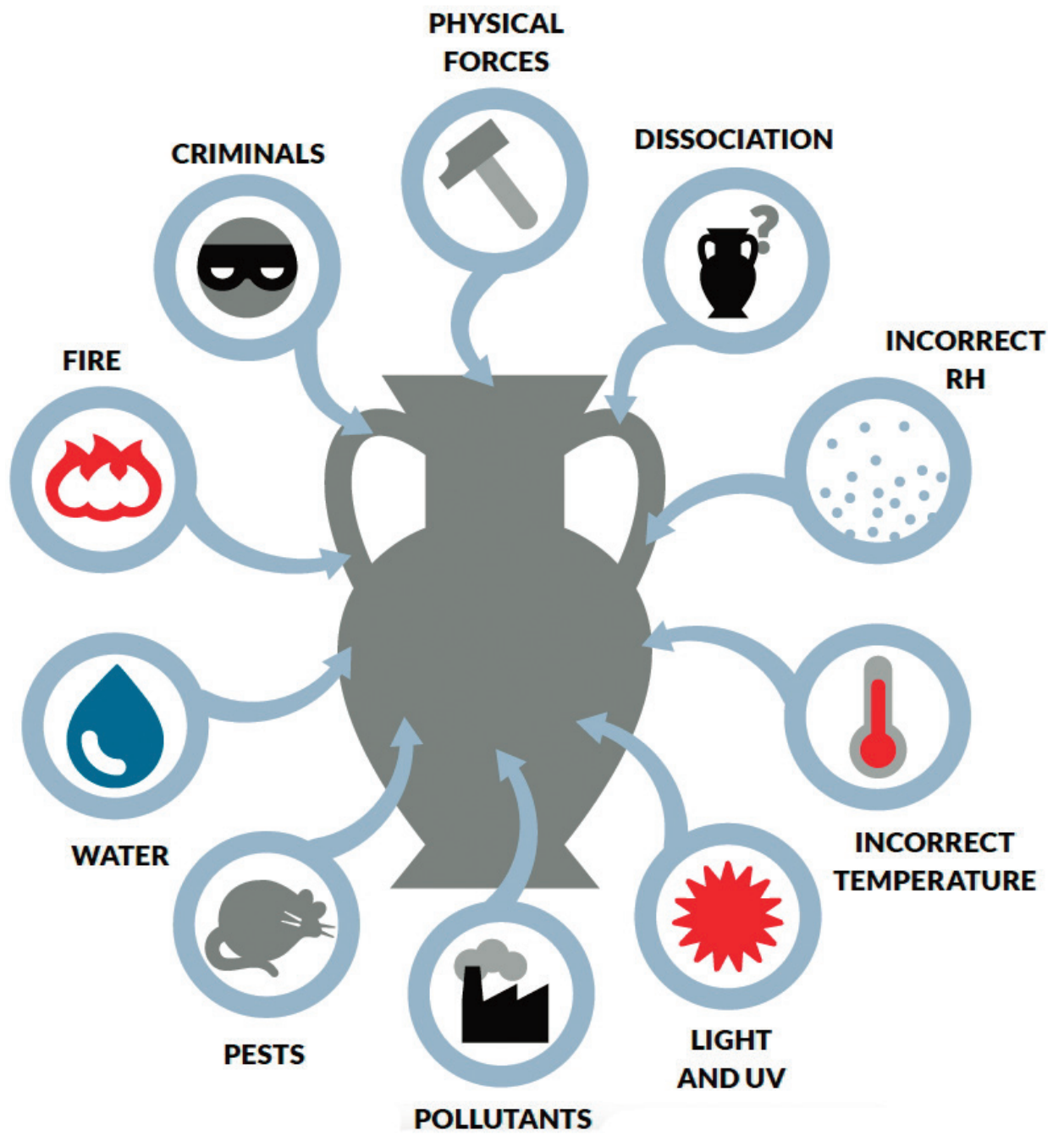

dentes o negligencia humana. Gran parte de los custodios subestiman las probabilidades y los efectos de situaciones esporádicas, como por ejemplo, las filtraciones de agua. Existe una tendencia a utilizar los sótanos para almacenar colecciones y archivos y a dejar cajas con materiales en el suelo, quizás sólo ‘temporalmente'... (p. 11)

5. Pestes. Las pestes o plagas son circunstancias particulares en las que se presentan organismos vivos capaces de deformar, dañar y destruir los bienes culturales, que pueden ocasionar la pérdida parcial de un bien. Strang \& Kigawa (2009) señalan al respecto lo siguiente:

En la medida en que han aumentado los lugares ocupados por los seres humanos, así como sus actividades agrícolas, innumerables plagas se han comenzado a adaptar, encontrando nichos en nuestros edificios (...) Estas plagas se han esparcido por el mundo y han proliferado a través del comercio y del turismo.

Especies de microorganismos, insectos y roedores representan a la mayoría de las plagas que afectan al patrimonio cultural. (...) Otras plagas, como aves dormideras, moluscos (perforadores marinos), murciélagos, otros mamíferos invasivos, lagartos, etc., aunque no predominantes en las colecciones a nivel global, pueden significar importantes daños en lugares específicos.
Figura 2. Los diez agentes de deterioro y pérdida

Fuente: A Guide to Risk Management of Cultural Heritage (p. 27), por ICCROM, 2016, Sharjah, United Arab Emirates: Autor. 
Asimismo, de cara a construir una mirada de conjunto, es posible identificar la existencia de 830,075 especies definidas de insectos, 100,800 especies de hongos y 4,496 especies de mamíferos (Lecointre \& Le Guyader, 2006), aunque las plagas equivalgan solo a una fracción de dichas especies...

6. Contaminantes. Sobre estos agentes específicos, Tétreault (2009), señala lo siguiente:

....están agrupados dentro de un rango de compuestos que pueden presentar reacciones químicas con algún componente de un objeto. Estos pueden ser gases, aerosoles, líquidos o sólidos tanto de origen antropogénico como natural, todas consideradas sustancias que poseen efectos adversos sobre los objetos. Los depósitos de partículas sólidas se consideran contaminantes, y aunque no necesariamente causen daño, sí provocan alteración en las características estéticas de los objetos en cuestión. En algunos casos, las partículas finas depositadas en la superficie de un objeto pueden estar fuertemente adheridas.

Existen tres modalidades de acción por parte de los contaminantes para llegar a un objeto y provocar deterioro en un museo. En la primera, los contaminantes son transportados por el aire; en la segunda son transferidos entre dos materiales en contacto; y en la tercera, son intrínsecos, es decir ya existen como parte del material que compone el objeto, o se forman durante reacciones químicas sobre o dentro de éste, y se denominan contaminantes secundarios. (p. 13)

7. Luz y UV. La exposición a la luz y los rayos UV, especialmente durante periodos de exposición no controlados, también pueden ser un factor de deterioro. Por ejemplo, puede provocar la pérdida de coloración en una capa pictórica, lo que constituye la pérdida parcial de un bien. Según Michalski,

...se plantea la necesidad de lograr el equilibrio entre la adecuada visualización de los bienes exhibidos y la minimización del daño generado por la luz, sin dejar de lado el principio ético que busca el equilibrio entre los derechos de nuestra propia generación, con los de aquellas que están por venir. En la práctica, esto implica considerar las necesidades y características de los objetos, ya que la sensibilidad a la luz y los requerimientos de visualización son variables. Asimismo, es preciso considerar que muchos museos cuentan con una iluminación variable y deficientemente controlada. (2009a, p. 15)

8. Temperatura incorrecta. A diferencia del fuego, las filtraciones de agua y la aparición de plagas, la temperatura no se considera un agente de deterioro, porque solo puede regularse. Técnicamente, la temperatura incorrecta es un obstáculo para conservación del patrimonio cultural; por ende, es considerada un riesgo, como señala Michalski (2009b). En la práctica, es posible establecer tres categorías de temperaturas incorrectas:

- Temperatura muy alta: Esta categoría puede subdividirse en aquellas ocasionadas por fenómenos químicos, físicos o biológicos. El primer caso es el más relevante para museos y archivos, ya que las temperaturas normales en una habitación son demasiado altas para la preservación a largo plazo de materiales inestables manufacturados por el hombre, especialmente aquellos que sirven como soporte de imágenes, sonido y texto. De hecho, en la mayoría de museos solamente tales colecciones de archivo reciben alguna atención especial en cuanto a la regulación de la temperatura.

- Temperatura muy baja: En general, las temperaturas bajas son beneficiosas para la conservación de las colecciones antes mencionadas. Sin embargo, algunos objetos que incluyen polímeros, como las pinturas, se vuelven más quebradizos y frágiles. Afortunadamente, una manipulación cuidadosa puede mitigar la mayoría de los riesgos.

- Fluctuación de temperatura: Este fenómeno es el de más compleja resolución, y genera un sinnúmero de solicitudes de control climático (junto con la preocupación por las fluctuaciones en la humedad relativa). No obstante, el énfasis en las fluctuaciones de temperatura ha sido desproporcionado en relación a su importancia para la preservación de las colecciones. 


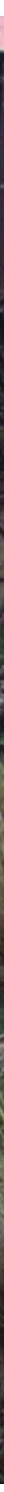

9. Humedad relativa incorrecta. La humedad relativa (HR), al igual que la temperatura, no puede considerarse un agente de deterioro, pues no es posible obviarla:

...es una medida de lo que cotidianamente llamamos 'humedad'. Es aquella característica del aire que oscila entre húmedo y seco. En realidad, no percibimos la HR por sí misma, sino que percibimos la humedad o sequedad de nuestros cuerpos en reacción a la HR ambiente, o asimismo, percibimos el efecto sobre los materiales tales como el papel o la tela, los que se vuelven húmedos o secos como respuesta a la HR. Aunque podemos sentir la diferencia entre ambos extremos, para todos los propósitos prácticos necesitaremos confiar en instrumentos que nos informen qué tipo de HR existe (...).

Desde una perspectiva práctica de evaluación de riesgos, las variadas formas de HR incorrecta pueden subdividirse en 4 tipos:

- Humedad, sobre un $75 \%$ de HR

- HR sobre o bajo un índice crítico para determinado objeto
Figura 3. Fotografía del deterioro de la estructuras de la mina de Mina de San Bárbara, expuestas a la intemperie. Comunidad de Santa Bárbara, Huancavelica, Perú

Fuente: Registro de Clary Santander, setiembre de 2013. 


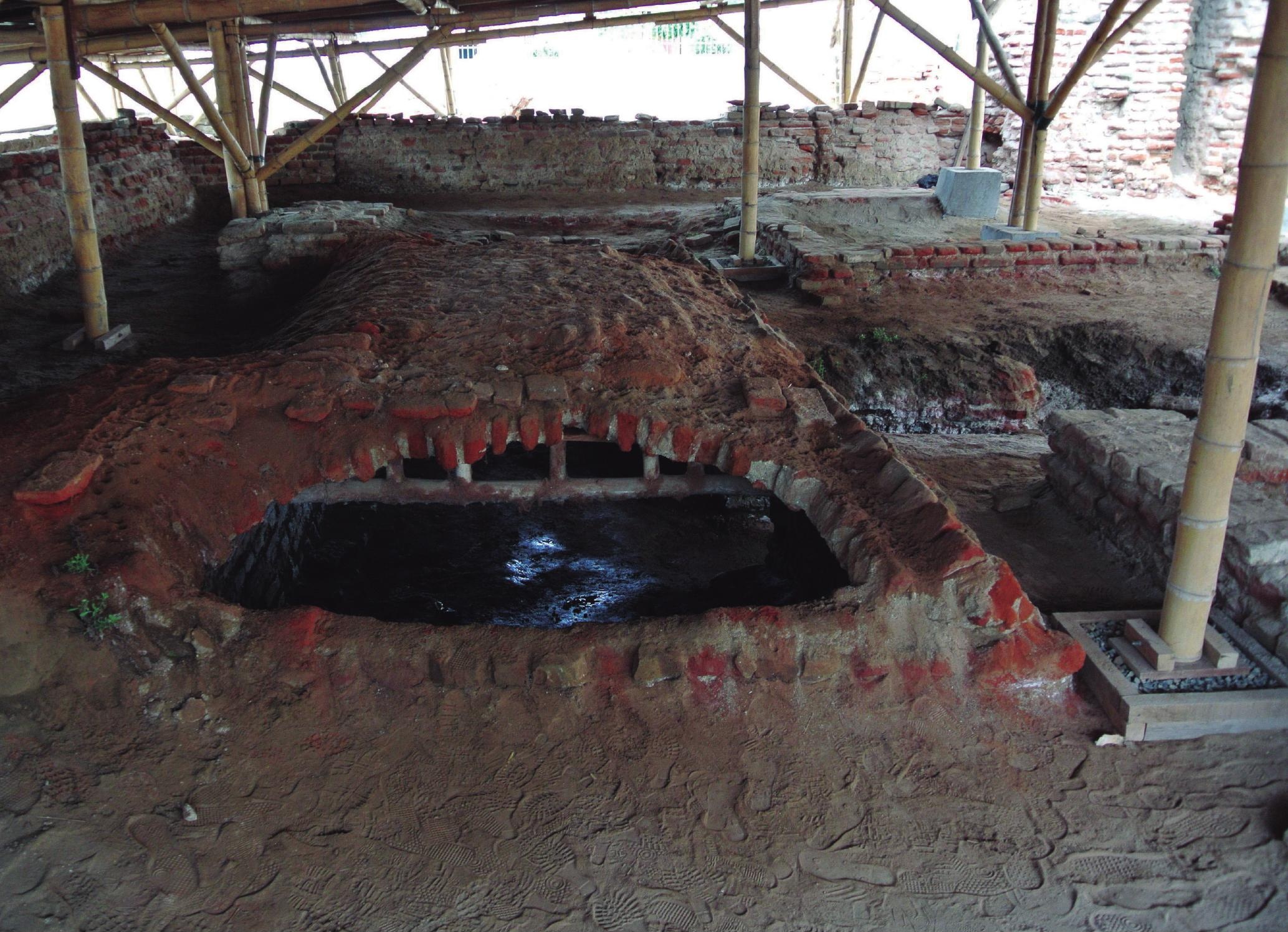

Figura 4. Fotografía del deterioro de la cripta del Templo de San Francisco, expuesta a la humedad. Zaña, Lambayeque, Perú

Fuente: Registro de Clary Santander, julio de 2015.
- HR sobre $0 \%$

- Fluctuaciones de HR (Michalski, 2009c, p. 15)

10. Disociación. Al respecto de este singular agente de deterioro, Robert \& Paisley (2009) señalan lo siguiente:

- La disociación surge de la tendencia natural de los sistemas ordenados a deshacerse a lo largo del tiempo. Para prevenirla, es necesario modificar los procesos de mantenimiento y otras barreras. La disociación provoca la pérdida de objetos, de su información relacionada o de la capacidad para recuperar o asociar objetos e información. Este agente puede manifestarse como:

- Eventos únicos, poco comunes y catastróficos; que provocan importantes pérdidas de información, de objetos o del valor de éstos.

- Eventos esporádicos y graves que ocurren cada ciertos años o décadas, provocando la pérdida de información, de objetos o del valor de éstos.

- Eventos o procesos continuos que provocan la pérdida de información, de objetos o del valor de éstos.

A diferencia de los otros nueve agentes de deterioro que afectan principalmente el estado físico de los objetos, la disociación incide tanto en los aspectos legales como intelectuales y/o culturales de un objeto, pudiéndose considerar como un agente metafísico. Otra caracterís- 


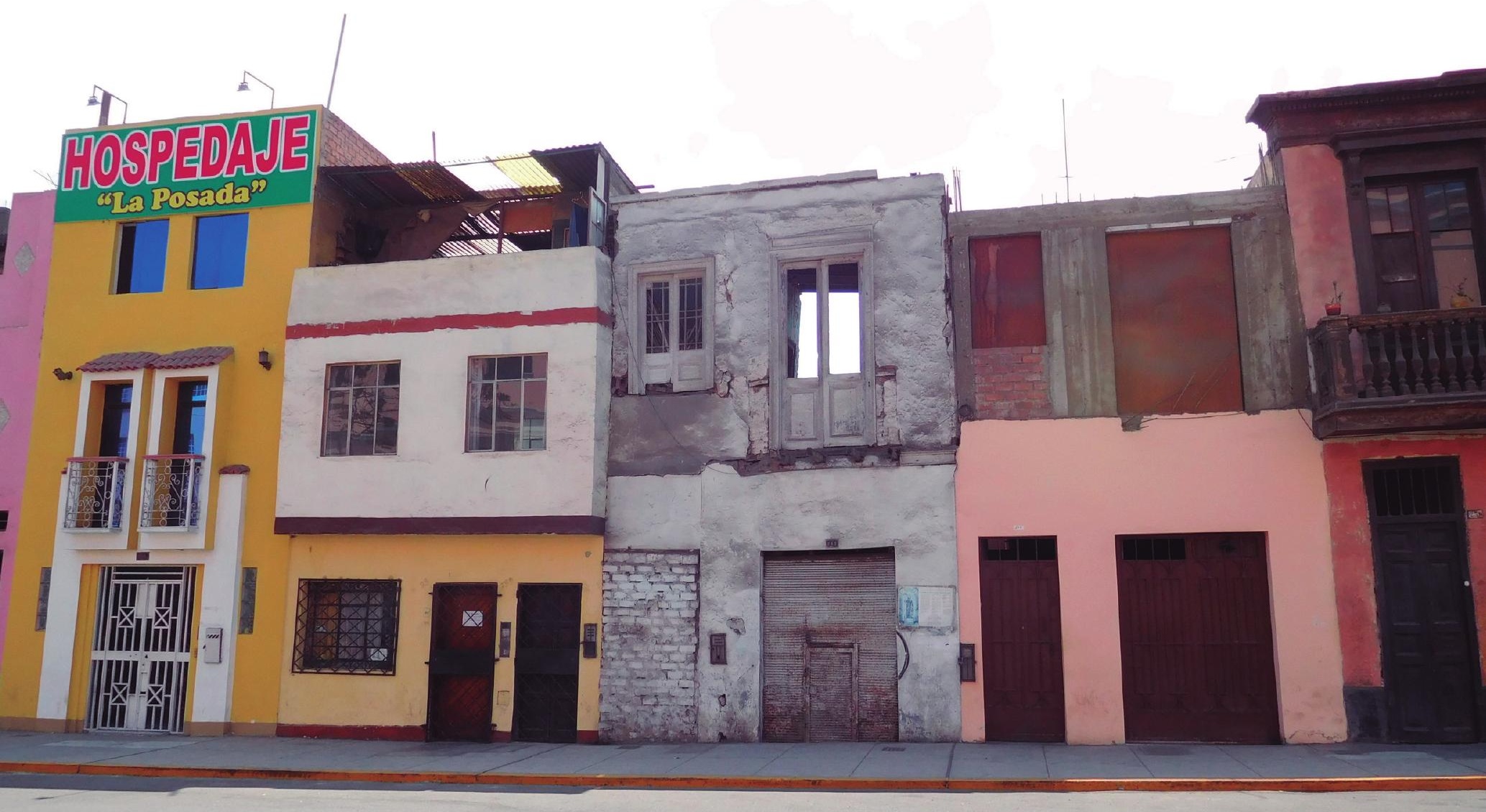

tica única de este agente, consiste en que la pérdida de valor de uno o unos cuantos objetos dentro de una colección, puede afectar el valor de todo el conjunto. Es por esto que debe considerarse el efecto de la presencia de objetos mezclados en los grupos de muestra. (...). Si un investigador, biólogo, arqueólogo o historiador observa una cantidad pequeña, pero significativa de objetos provenientes de diferentes grupos que están mezclados entre sí, la colección completa se considera comprometida; por consiguiente, unas pocas muestras cruzadas, pueden lograr que una colección pierda buena parte de su valor como referencia y para la investigación. (p. 18)

A nivel urbano, este agente puede ejemplificarse en la pérdida de bienes inmuebles, intervenciones inadecuadas que generan la alteración del perfil urbano, demoliciones clandestinas de edificaciones e intervenciones fuera del marco normativo, las cuales pueden llevar a la pérdida total o parcial de un bien (Figuras 3, 4, 5 y 6).

\section{Los tres tipos de ocurrencia}

Según Michalski \& Pedersoli, las "amenazas pueden dividirse en dos grandes grupos principales: eventos y procesos continuos" (2011, p. 42). Para fines prácticos, es importante considerar tres tipos de ocurrencia de riesgo: a) eventos extraordinarios, b) eventos frecuentes y c) procesos continuos (ICCROM, 2016). Cuando los riesgos son la consecuencia de eventos puntuales, se intenta establecer la frecuencia con que la que estos se producen; cuando son procesos, se trata de estimar la velocidad con la que el daño se acumula (ICCROM, 2016).
Figura 5. Fotografía de un tramo alterado del perfil urbano del Jr. Madera del Paseo de Aguas. Rímac, Lima, Perú

Fuente: Registro de la Clary Santander, abril de 2016. 

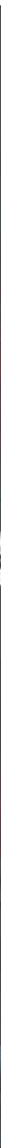

Figura 6. Incendio producido del 16 de setiembre de 2016 en el Templo de San Sebastián. Cusco, Perú

Fuente: Recuperado de http://diariocorreo.pe/peru/cusco-incendio-destruye-el-80-de-la-iglesia-san-sebas tian-698645/
1. Eventos extraordinarios. "(...) ocurren con una frecuencia menor a uno de cada 100 años (...). Como resultado los eventos extraordinarios no son parte de la experiencia directa de la mayoría del personal de [una organización]" (Michalski \& Pedersoli, 2011, p. 42). En el caso del patrimonio edificado, tales eventos incluyen inundaciones, sismos e incendios de gran escala, entre otros.

2. Eventos frecuentes. “(...) pueden ocurrir muchas veces en un siglo. Estos eventos son parte de la experiencia directa [del personal de una organización]" (Michalski \& Pedersoli, 2011, p. 42). En lo que respecta al patrimonio inmueble, este tipo de eventos incluyen filtraciones de agua, sismos e incendios de poca envergadura, colapsos parciales, demoliciones clandestinas, robo de componentes, entre otros.

3. Procesos continuos. “(...) pueden ocurrir continua e intermitentemente. A través de los años" (Michalski \& Pedersoli, 2011, p. 42). Las personas vinculadas a los bienes observan cotidianamente el efecto acumulativo del daño. En el caso del patrimonio edificado, cabe mencionar entre tales procesos el envejecimiento propio de los materiales: la erosión de la piedra, la corrosión de los metales, el desvanecimiento de algunos colores, el desgaste, la acumulación de smog, entre otros.

\section{Las escalas $A B C$ para el análisis de riesgos}

Según señalan Mattos \& Gonçalves, “Existen diferentes metodologías para el cálculo de la magnitud de los riesgos que, aun utilizando diferentes escalas numéricas, conducen a la misma interpretación de los resultados" (2015, p. 145). El método de escalas numéricas, llamado $A B C$, se emplea para cuantificar la frecuencia o tasa de ocurrencia, 
y la pérdida esperada de valor para los diferentes riesgos (ICCROM, 2016). Las escalas $A B C$ tienen tres componentes: el componente $A$, denominado "frecuencia o tasa"; el componente B, denominado "pérdida a futuro de cada bien afecto", y el C, el valor actual de los todos los bienes afectados" (ICCROM, 2016, p. 76) (Figura 7).

El componente " $\mathrm{A}$ ", frecuencia o tasa, "es uno sólo (...) aunque mezcla dos tipos de momentos: eventos y procesos" (Michalski \& Pedersoli, 2011, p. 54). En el caso de un evento, este componente indica la frecuencia con la que se espera que este ocurra, es decir, el tiempo promedio entre dos ocurrencias del mismo, mientras que cuando se trata de procesos continuos, este componente indica cuánto tiempo tomará que se produzca una pérdida específica (ICCROM, 2016).

El componente " $\mathrm{B}$ ", pérdida a futuro de cada bien afectado, indica la magnitud de la pérdida de valor de cada bien afectado. Para estimar tal pérdida, es necesario visualizar el tipo y la magnitud del daño que sufrirán (ICCROM, 2016). La pérdida de valor del bien puede ser total o despreciable.

El componente " $\mathrm{C}$ ", valor actual de todos los bienes afectados, señala cuánto del valor del patrimonio se ve afectado por el riesgo (ICCROM, 2016). Por ejemplo, qué relevancia tiene la pérdida total de un edificio patrimonial producto de un incendio, demolición u otro fenómeno en relación al total de bienes patrimoniales, al total del patrimonio inmueble edificado (Figura 7).

Una vez calculados los tres componentes, se puede obtener la magnitud de riesgo (MR), es decir, el potencial de pérdida del valor del patrimonio.

\section{Reflexión final}

Son muchos los retos que deben enfrentarse para determinar qué caminos debe tomar la gestión de riesgos del patrimonio cultural. En ese contexto, el presente artículo tiene entre sus fines el motivar investigaciones similares. Si no se logra incluir la gestión de riesgos como parte de la gestión del patrimonio cultural, ello derivará en un grave deterioro en el caso del patrimonio histórico inmueble dada su vulnerabilidad ante procesos de deterioro continuo. Asimismo, debe tenerse en cuenta que la mayor parte de las edificaciones patrimoniales se encuentran construidas con técnicas tradicionales, en materiales orgánicos como tierra, piedra y madera, por lo que son especialmente vulnerables a fenómenos naturales como sismos, inundaciones y eventos provocados por causas externas, como incendios, intervenciones inadecuadas, demoliciones, entre otros. Otro aspecto que debe tomarse en cuenta son los pocos recursos destinados a la conservación del patrimonio cultural inmueble, a lo cual se suma la limitada incorporación de conocimientos científicos y técnicos en su respectiva gestión. En este contexto, resulta importante orientar adecuadamente el destino de los recursos disponibles, por lo que valorar los riesgos y cuantificar la magnitud es un proceso crucial para priorizar acciones y dirigir la inversión, tanto pública como privada.

Los últimos acontecimientos (verano de 2017 en Perú), producto del denominado fenómeno El Niño, han implicado la reanudación de las discusiones y cuestionamientos
Figura 7. Magnitud de riesgo Fuente: A Guide to Risk Management of Cultural Heritage (p. 76), por ICCROM, 2016, Sharjah, United Arab Emirates: Autor. 
sobre los riesgos y la planificación. Sorprendentemente, gracias a la difusión de diversos medios de comunicación, fue posible observar cómo el Centro Histórico de Trujillo y Piura quedaron inundados por los efectos de las lluvias y los huaycos. La situación fue similar en otros espacios urbanos de valor cultural, como la zona monumental de Chosica y Chiclayo. Sin embargo, no es la primera vez que se suscitan catástrofes similares: otro evento importante a nivel urbano lo constituyó el incendio ocurrido el 29 de diciembre de 2001 en el sector denominado Mesa Redonda, en el Centro Histórico de Lima, el cual según Arce (2001)

...causó la muerte de 277 personas, 247 resultaron heridos de los cuales 137 sufrieron quemaduras, 45 asfixia y 38 politraumatismos, se reportaron 180 desaparecidos, las pérdidas materiales alcanzaron unos diez millones de US dólares. En la zona central del incendio se habrían producido temperaturas de hasta $1200^{\circ} \mathrm{C}$ lo cual carbonizó a las víctimas, una bola de fuego de $800^{\circ} \mathrm{C}$ se desplazó por las calles atrapando personas y vehículos. (p. 120)

Además, el incendio tuvo otros impactos negativos en el área urbana de Mesa Redon$\mathrm{da}$, que perdió varias edificaciones declaradas patrimonio.

Otro evento considerable fue el sismo de 2001, que afectó gravemente el Centro Histórico de Arequipa, según señala la Municipalidad de la misma ciudad (2002):

Producido el terremoto de grado 6.9 en la escala de Richter, (...) el centro histórico resultó gravemente afectado (...). Se ofrecieron 130 voluntarios, ingenieros y arquitectos, organizados en brigadas de apoyo (...). Se evaluó 1105 predios que equivalen al 44\% de la totalidad de inmuebles ubicados en el centro histórico... (p. 56)

Al parecer, solo los eventos extraordinarios constituyen una llamada de atención y alarma para la sociedad en general. Sin embargo, los efectos de los procesos continuos, que son los que con mayor frecuencia se suscitan, también requieren acciones planificadas para mitigarlos. Ante esta situación, un enfoque integrador promueve acciones a muchas escalas con enfoques diversos.

\section{Referencias}

Arce, J. (2001). Grandes incendios urbanos: Mesa Redonda, Lima 2001. En Actas del Simposio: Emergencias y desastres.

Ballart, J. \& Juan Tresserras, J. (2001). Gestión del patrimonio cultural. Barcelona, España: Ariel.

Centro Internacional de Estudios para la Conservación y la Restauración de los Bienes Culturales (2016). A guide to risk management of cultural heritage. Sharjah, Emiratos Árabes Unidos: Canadian Conservation Institute.

Congreso de la República de Perú (2004). Ley № 28296: Ley General del Patrimonio Cultural de la Nación. En Diario Oficial El Peruano, 22 de julio de 2004. Perú.

Gobierno Peruano (2011). Ley 29664: Ley que crea el Sistema Nacional de Gestión del Riesgo de Desastres (SINAGERD).

González-Varas, I. (2012). Patrimonio cultural: Conceptos debates y problemas. Madrid, España: Cátedra.

Hayakawa, J. (2009). Gestión del Patrimonio Cultural y centros históricos latinoamericanos: Tendiendo puentes entre el patrimonio y la ciudad. Lima, Perú: Universidad Nacional de Ingeniería.

Instituto Colombiano de Normas Técnicas y Certificación. (2004). Norma Técnica Colombiana NTC 5254. Gestión del riesgo.

Jorquera, N. (2014). Método integral de evaluación del riesgo sísmico del patrimonio arquitectónico menor. Apuntes, 27(1), 52-63.

Marcon, P. (2009). Fuerzas físicas [Documento inédito del Curso Internacional de Gestión de Riesgos para el Patrimonio Cultural, edición en español desarrollada por ICCROM]. Canadian Conservation Institute, Canadá. 
Mattos-Cárdenas, L. \& Gonçalves, W. B. (2015). Apuntes sobre la terminología de la gestión de riesgos aplicada a la conservación del patrimonio cultural en el contexto latinoamericano. En $\checkmark$ Congreso chileno de Conservación y Restauración, desarrollado en la Asociación Gremial de Conservadores de Chile, Chile. Recuperado de https://www.academia.edu/21924910/2015_Apuntes_sobre_la_terminolog\%C3\%ADa_de_la_Gestión_de_Riesgos_aplicada_a_la_conservación_del_Património_Cultural_en_el_contexto_latinoaamericano

Michalski, S. \& Pedersoli, J. (2011). Manual de referencia para el Método de Gestión de Riesgos del ICC-ICCRON-RCE [Documento inédito del Curso Internacional de Gestión de Riesgos para el Patrimonio Cultural, traducción libre de la autora al español, 2013].

Michalski, S. (2009a). Luz visible, radiación ultravioleta e infrarroja [Documento inédito del Curso Internacional de Gestión de Riesgos para el Patrimonio Cultural, edición en español desarrollada por ICCROM]. Canadian Conservation Institute, Canadá.

Michalski, S. (2009b). Temperatura incorrecta [Documento inédito del Curso Internacional de Gestión de Riesgos para el Patrimonio Cultural, edición en español desarrollada por ICCROM]. Canadian Conservation Institute, Canadá.

Michalski, S. (2009c). Humedad Relativa Incorrecta. [Documento inédito del Curso Internacional de Gestión de Riesgos para el Patrimonio Cultural, edición en español desarrollada por ICCROM]. Canadian Conservation Institute, Canadá.

Ministerio de Cultura (2016). Patrimonio histórico inmueble. Recuperado el 23 de setiembre de 2016 de http://www.cultura.gob.pe/es/serviciosenlinea/patrimoniohistoricoinmueble

Ministerio de Cultura (2016). Reporte bienes históricos declarados. Recuperado el 23 de setiembre de 2016 de http://www.infocultura.cultura.pe/infocultura/\#

Ministerio de Educación (2006). Reglamento de la Ley № 28296 aprobado por Decreto Supremo № 011-2006-ED. Diario Oficial El Peruano, 2 de junio de 2006. Perú.

Municipalidad de Arequipa (2002). Plan Maestro del Centro Histórico de Arequipa: Vol. 1. Diagnóstico. Recuperado de http://www.cultura.gob.pe/sites/default/files/paginternas/tablaarchivos/04/vol1diagnostico.pdf

Oficina de las Naciones Unidas para la Reducción del Riesgo de Desastres. (2009). Terminología sobre reducción de riesgo de desastres. Recuperado el 15 de septiembre de 2016 de http:// www.unisdr.org/files/7817_UNISDRTerminologySpanish.pdf

Organización de las Naciones Unidas para la Educación, la Ciencia y la Cultura. (2008). Carpeta de información sobre el Patrimonio Mundial. Centro del Patrimonio Mundial. Francia. Recuperado el 15 de septiembre de 2016 de https:/www.google.com/url?sa=t\&rct=j\&$\mathrm{q}=\&$ esrc $=$ s\&source $=$ web\& $c d=1$ \&ved=0ahUKEwib6uGrgt7PAhUGHT4KHc62C34QFgge MAA\&url=http\%3A\%2F\%2Fwhc.unesco.org\%2Fdocument\%2F102074\&usg=AFQjCNES8LSGZnVOPu9CZRPXDf9bJMKdbg

Organización de las Naciones Unidas para la Educación, la Ciencia y la Cultura. (2014a). Gestión del riesgo de desastres para el Patrimonio Mundial. Recuperado el 25 de setiembre de 2016 de http://unesdoc.unesco.org/images/0022/002281/228134s.pdf

Organización de las Naciones Unidas para la Educación, la Ciencia y la Cultura. (2014b). Gestión del patrimonio mundial cultural. Centro de Patrimonio Mundial de la UNESCO. Francia. Recuperado el 25 de noviembre de 2016 de http://whc.unesco.org/document/130490

Organización de las Naciones Unidas para la Educación, la Ciencia y la Cultura. (2014c). Indicadores UNESCO de Cultura para el desarrollo. Francia. Recuperado el 25 de noviembre de 2016 de http://es.unesco.org/creativity/sites/creativity/files/iucd_manual_metodologico_1.pdf

Robert, R. \& Paisley, S. (2009). Disociación [Documento inédito del Curso Internacional de Gestión de Riesgos para el Patrimonio Cultural, edición en español desarrollada por ICCROM]. Canadian Conservation Institute, Canadá.

Stewart, D. (2009). Fuego [Documento inédito del Curso Internacional de Gestión de Riesgos para el Patrimonio Cultural, edición en español desarrollada por ICCROM]. Canadian Conservation Institute, Canadá. 
devenir VOL. 4, N7, ENERO - JUNIO 2017, PP. 145-162 - ESTUDIOS | ISSN 2312-7562

UNIVERSIDAD NACIONAL DE INGENIERÍA, LIMA

Strang, T. \& Kigawa, R. (2009). Combatiendo las plagas del patrimonio cultural [Documento inédito del Curso Internacional de Gestión de Riesgos para el Patrimonio Cultural, edición en español desarrollada por ICCROM]. Canadian Conservation Institute, Canadá.

Tello, S. (2002). En Torno al Patrimonio e Interdisciplinariedad. En III Encuentro Iberoamericano Fórum UNESCO, Universidad y Patrimonio. Universidad de San Martín de Porres, Lima, Perú.

Tétreault, J. (2009). Contaminantes. [Documento inédito del Curso Internacional de Gestión de Riesgos para el Patrimonio Cultural, edición en español desarrollada por ICCROM].

Tremain, D. (2009a). Robo y vandalismo. [Documento inédito del Curso Internacional de Gestión de Riesgos para el Patrimonio Cultural, edición en español desarrollada por ICCROM].

Tremain, D. (2009b). Agua. [Documento inédito del Curso Internacional de Gestión de Riesgos para el Patrimonio Cultural, edición en español desarrollada por ICCROM]. 